\title{
Expression Analysis using kernel Extreme Learning Machines
}

\author{
Ahmed Farhad, Javed Khan
}

\section{INTRODUCTION}

Expression analysis is a topic covered under affective computing which is under a lot of research in the field of computer vision. We propose an expression analysis algorithm that utilizes kernel ELMs and CNNs to determine the state of the expression. The expressions include sadness, happiness, fear, anger, disgust, surprise and neutral. The first step is to detect the face in the image and to do that we use the DPM face detector and for extracting the features we use the VGG face network. Once we have the features of the face selected in the image we use kernel extreme learning machines (ELM) due to it's high speed of execution and the accuracy. Seven ELMs are needed to obtain predictors for seven expressions. The prediction is then performed using a fusion network that obtains features from two independent networks. Along with the input from the two networks, scores are taken from all the ELM models as input, to enhance accuracy.

\section{REFERENCES}

[1] Gowda, S.N., 2017. Human activity recognition using combinatorial Deep Belief Networks. In Proceedings of the IEEE Conference on Computer Vision and Pattern Recognition Workshops (pp. 1-6).

[2] Wang, H. and Schmid, C., 2013. Action recognition with improved trajectories. In Proceedings of the IEEE international conference on computer vision (pp. 3551-3558).

[3] Jhuang, H., Gall, J., Zuffi, S., Schmid, C. and Black, M.J., 2013. Towards understanding action recognition. In Proceedings of the IEEE international conference on computer vision (pp. 3192-3199).

[4] Gowda, S.N. and Yuan, C., 2018, December. ColorNet: Investigating the importance of color spaces for image classification. In Asian Conference on Computer Vision (pp. 581-596). Springer, Cham.

[5] Li, W., Zhang, Z. and Liu, Z., 2010, June. Action recognition based on a bag of 3d points. In 2010 IEEE Computer Society Conference on Computer Vision and Pattern Recognition-Workshops (pp. 9-14). IEEE.

[6] Gowda, S.N., 2016, December. Age estimation by LS-SVM regression on facial images. In International Symposium on Visual Computing (pp. 370-379). Springer, Cham.

[7] Gowda, S.N., 2016, November. Fiducial points detection of a face using RBF-SVM and adaboost classification. In Asian Conference on Computer Vision (pp. 590-598). Springer, Cham. 